\title{
A 3D-printed open access photoreactor designed for versatile applications in photoredox- \& photoelectrochemical synthesis
}

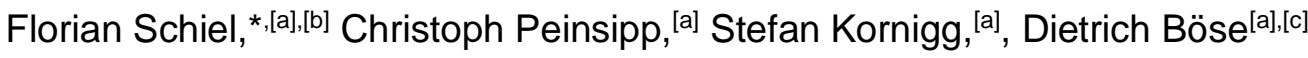

Supporting information contains the equipment list and constructing manual. All 3D-printing files are freely available and if Fusion 360 files are wanted, please contact Christoph Peinsipp (christoph.peinsipp@boehringer-ingelheim.com)

\begin{abstract}
Most published photochemical reactions are still not performed under standardized conditions. It is well known that the control of light intensity, the exact reaction temperature and other parameters are crucial for the success of a photochemical reaction. However, for most reactions reported in the literature, these parameters are not precisely controlled and recorded. As a result, the reproduction of these reactions is difficult and the progress in the field of photoredox chemistry is hampered by this limitation. To address this problem, a 3D-printed photoreactor was designed which can be easily replicated with a small number of inexpensive and easily available components. Equipped with thermoelectric coolers, the reactor can access and precisely control the temperature in the range of $-17^{\circ} \mathrm{C}$ to $80^{\circ} \mathrm{C}$, while reactions under high-intensity irradiation are performed with LED lamps from Kessil or HepatoChem. The practical design of the vial holder allows a versatile use of different reaction vessels - in addition to fast reaction optimization with up to eight vials simultaneously, upscaling in batch and flow is easily possible. Due to the high light intensity, the catalyst loading can be reduced to 0.1 mol\% for large-scale reactions. The flexibility of the vial holder is demonstrated by combining IKA's ElectraSyn 2.0 with the photoreactor to perform photoelectrochemical reactions in a reproducible manner.
\end{abstract}

\section{Introduction}

The use of visible light as an alternative energy source to drive chemical reactions has enabled the development of novel and unique transformations over the last decade. With the growing interest, many groups have diversified their research to the field of synthetic photochemistry and numerous experimental setups were created. However, the large variety of setups and light sources are problematic with regards to reproducibility, since the outcome of the reaction depends on many factors of which some are not fully understood. ${ }^{[1]}$ Oftentimes, little or no reactor/setup details are reported and therefore it may be challenging to reproduce the published experimental results. ${ }^{[2]}$ To address this issue a standardized set of equipment is needed, which must meet several requirements to be used on a broad basis (Figure 1).

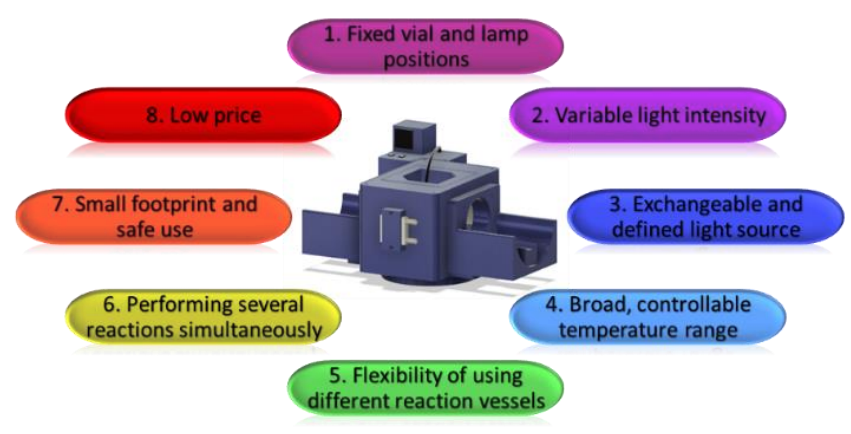

Figure 1. Summary of features a standardized photoreactor should fulfill to cover a broad application area.

1) Fixed vial and lamp position: A set distance between the light sources and the reaction vials enables constant light intensity in the reaction medium to ensure reproducibility.

2) Variable light intensity: Photochemical reactions are initiated by the absorption of photons and the effectiveness of the processes is determined by quantum yields. The kinetics of those processes are light intensity-dependent and therefore the reaction rate is affected by a change in the number of photons. Reactions with a low photoredox catalyst concentration are saturated already at lower irradiation while for higher catalyst loadings, or for direct irradiation of the reactants higher intensities are required. ${ }^{[3]}$ In several photoredox reactions a high photon flux showed a significant improvement in the transformation compared to the unsaturated variant, but sometimes it can cause a diminished yield and reproducibility problems. ${ }^{[4]}$ In some cases the chemoselectivity can be altered by changing the number of photons which are absorbed. ${ }^{[5]}$ Furthermore, according to the Beer-Lambert law, the light intensity decreases exponentially with depth of the reaction medium, which may decrease the efficiency of the process, especially for larger scale reactions. ${ }^{[3 \mathrm{~b}]}$

3) Exchangeable and defined light sources: In terms of selectivity and reproducibility, it is important to know and control the wavelength that drives the reactions. Light sources such as CFL or medium pressure $\mathrm{Hg}$ lamp cover a broad range of different emission bands and therefore the use of LEDs are currently the preferred choice due to the narrower emission bands. ${ }^{[2,3 b, 6]}$ On 
the other hand, the choice of the wavelength is also crucial to obtain an effective irradiation of the chromophore and to avoid side-reactions. ${ }^{[7]}$

4) Broad, controllable temperature range: Most photochemical reactions are carried out at ambient temperature since energy is introduced by the absorbed photons and often no or little temperature influence is observed. But if the photon absorption process is no longer the rate determining step, which is achieved at high light intensity, then the reaction is affected by the temperature. ${ }^{[3]}$ In addition, the generated intermediates are normally in the ground state, and temperature effects may arise due to the intrinsic activation barriers. ${ }^{[8]}$ On the other hand, some reactions require temperatures below $0{ }^{\circ} \mathrm{C} .{ }^{[9]}$ This shows that a widely accessible temperature range is essential for the best possible reaction optimization and the reproduction of literature examples.

5) Flexibility in the use of different types of vials and the possibility of upscaling (batch or flow): Different chemistries require different reaction vessels and therefore the vial holder should be easily adaptable to ensure that the reactions are reproducible.

6) Performing several reactions simultaneously: Allows a rapid screening of reaction conditions and parallel synthesis.

7) Practicality in terms of size, accessories and safety: A frequently used device is preferably small, and no external devices are required. It should also be well sealed to avoid exposure of the environment to high energetic radiation.

8) Price: The device should be affordable, since most research groups require several reactors.

Several companies have already addressed the problem of standardization and created commercially available photoreactors. At least five different companies offer them, with prices ranging from $1.800 €$ to over $20.000 €$. ${ }^{[4 a, 7 b, 10]}$ However, none of the commercial reactors fulfill all criteria discussed. The more features a reactor covers, the more expensive it becomes. Their main field of application is in the chemical industry and a standardized design is rarely found in recent publications. Therefore, we have decided to design an open-access photoreactor to ensure that more photochemical reactions are performed in a standardized reactor. This assumes that the reactor is suitable for many different applications (i.e. it should cover all points mentioned above) and at the same time the cost and size should be reduced to a minimum (about $450 €$ without lamps). In addition, the barrier to reproduction must be minimized by a simple and robust construction.

\section{Results and Discussion}

\section{Reactor design}

We mainly focused on three parts: 1) 3D-printing ${ }^{[11]}$ to quickly design prototypes at low cost, 2) air as cooling medium to ensure high light intensity and 3) thermoelectric cooling elements (TECs) as inexpensive and precise temperature control units. TECs are heat pumps consisting of P-type (holes) and N-type (electrons) semiconductors which are able to transport heat from the cooler

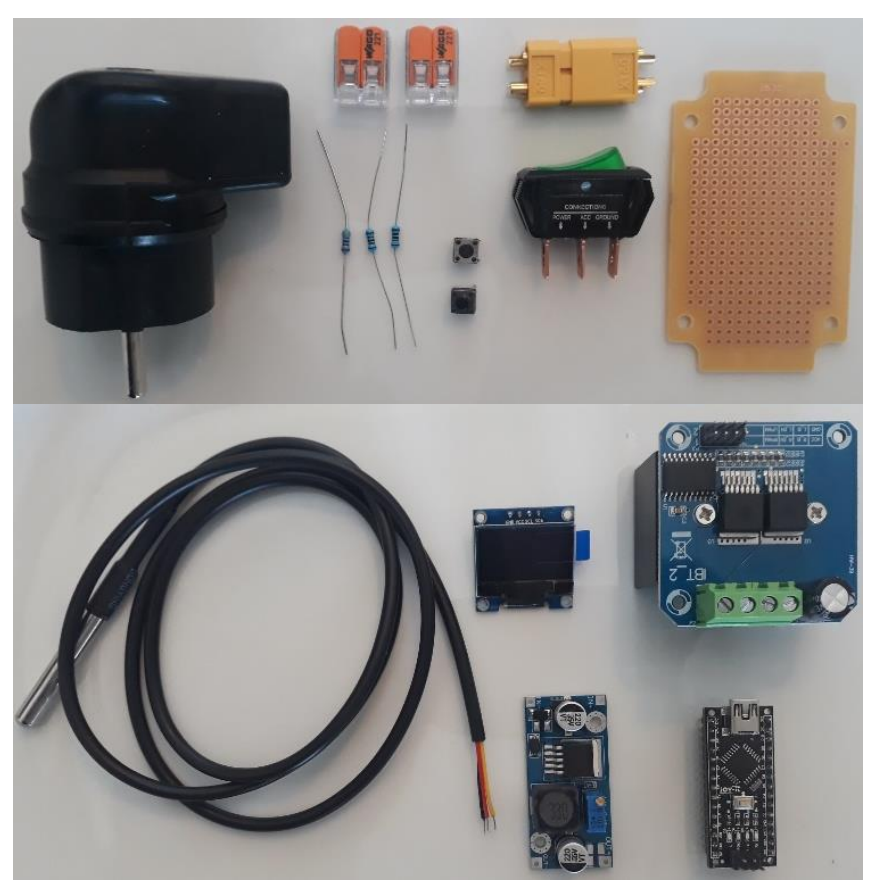

Figure 2. All electronic components that are required (except power supply unit and TECs). Upper picture (from left to right): Power plug, $2 \times$ connector clamp, $3 \times$ resistor, $2 \times$ pushbutton, XT60, On/Off switch, veroboard. Lower picture (from left to right): Temperature sensor, display, step down converter, motor driver, Arduino microchip.

to the hotter side by applying current. ${ }^{[12]}$ They are well suited for this purpose due to their small size and inexpensive availability. A reactor was then designed and 3D-printed with TECs inserted into the chamber walls (Figure 3). On the outside, the heat of the TECs is removed by an additional water-cooling circuit. Inside the reactor, heat transfer is achieved by heat sinks with a large surface area and by additional fans, which ensure optimal cooling of the entire chamber. The TECs are automatically switched off when the desired temperature is reached, the LED lamps heat up the chamber and the TECs switch on again. This process ensures that the temperature fluctuation of the air is kept within $\pm 1-3^{\circ} \mathrm{C}$, the fluctuation range inside of the reaction vessels is even smaller. The temperature range that can be used with this setup is between $-20^{\circ} \mathrm{C}$ and $+80^{\circ} \mathrm{C}$. The lower limit is determined by four factors: The thermal insulation, the efficiency of the TECs, the power of the fans and the light intensity. The thermal insulation is achieved by printing the reactor with $2 \mathrm{~cm}$ thick walls with $10 \%$ grid filling. This value indicates that the empty space is filled with $10 \%$ filament in 3D printing. However, the most important cooling factor is the efficiency of the TECs (including quality and performance). The chamber is cooled by two high-tech $8.5 \mathrm{~A}$ TECs to $-20^{\circ} \mathrm{C}$ without light. A temperature gradient is created in the reactor, with the coolest parts being the heat sinks, while the hottest area is in the beam of the lamps. How well the heat is dissipated is determined by the power of the fans. The most effective cooling is achieved by using high-speed fans at a voltage of 5-7 $\mathrm{V}$ (higher voltage reduces cooling efficiency and the noise level increases significantly). 

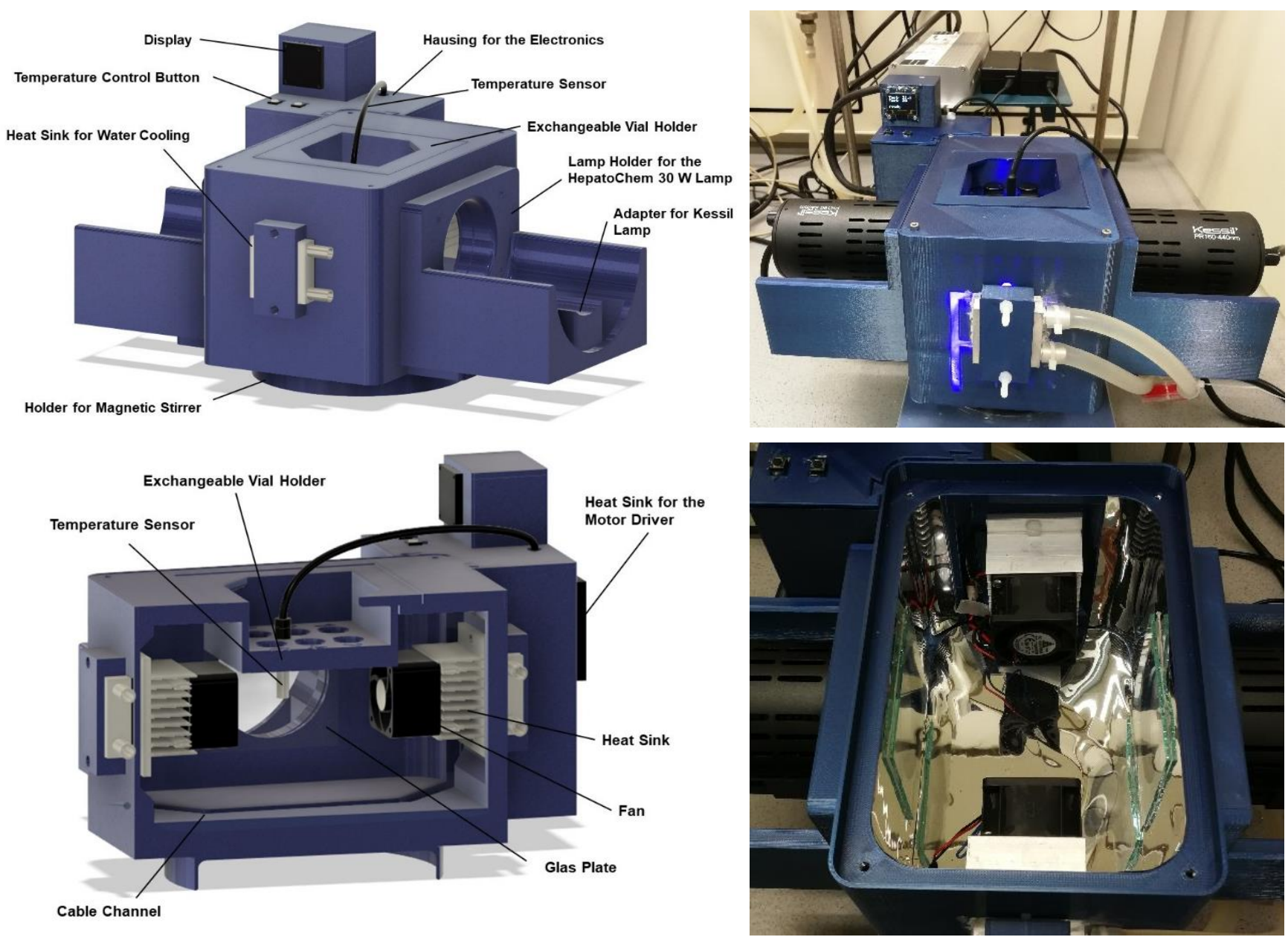

Figure 3. a) Reactor view from the top and b) from inside with all components labeled; c) Picture of the actual reactor where the LED lamps are turned on; d) Picture of the actual reactor from inside.

The effective working temperature is strongly dependent on the light intensity. While $-17^{\circ} \mathrm{C}$ is obtained with one Kessil PR160L $45 \mathrm{~W} 440 \mathrm{~nm}$ at $25 \%$ light intensity, $-2{ }^{\circ} \mathrm{C}$ can be reached with two Kessil PR160L 45 W 440 nm lamps at 100\% light intensity each. On the other hand, the upper temperature limit is defined by the glass transition point of the filament, which is $80^{\circ} \mathrm{C}$ for the used PETG. At this point a deformation of the reactor can occur. When working at temperatures below or near $0^{\circ} \mathrm{C}$, the potential problem of water condensation had to be addressed. Fortunately, it was found that no water condensation occurs on the reaction vessels. Instead, the water freezes on the heat sinks, which is reduced to a minimum when the reactor is sealed almost airtight. The closed system has the additional advantage that there is no light pollution of the environment, which allows a safe handling of the reactor environment with UV and intense blue light (Figure 3).

The electronic control unit is based on an Arduino microchip and a motor driver, to ensure that the reactor remains small, practical and easy to rebuild (Figure 2). The microchip controls the temperature in the chamber while the motor driver is responsible for switching the TECs on and off. The water-cooling for the TECs alone is sufficient to maintain the chamber around $40{ }^{\circ} \mathrm{C}$ (with two
Kessil PR160L 45 W 440 nm lamps, 100\% light intensity each) and to increase the temperature range the microchip is necessary. It enables a change in the current flow, which reverses the mode of action of the TECs, where the chamber is heated while the outside is cooled. In addition, safety related functions are added, such as an emergency shutdown of the thermoelectric coolers in case of overheating if the water-cooling is not turned on (for a complete list of safety features see $\mathrm{SI}$ ).

To ensure that the reactor is easily rebuilt, the commercially available LED lamps from Kessil and HepatoChem were chosen as light sources. Both companies provide LED lamps at different wavelengths (365 - $740 \mathrm{~nm}$ ) with a narrow and well-defined emission band. Additionally, the light intensity of the Kessil lamps is dimmable. The two independent lamp positions enable the possibility of using two different wavelengths at the same time. The exchangeable vial holder allows the use of a variety of vessels to perform reactions in different scales. Due to the simple design it can be easily adjusted if different vessels are required (e.g. a Schlenk flask). Holders are provided for $1 \mathrm{ml}$ vials (HPLC vials), $4 \mathrm{ml}$ and $8 \mathrm{ml}$ vials, so that several reactions can be performed simultaneously. 


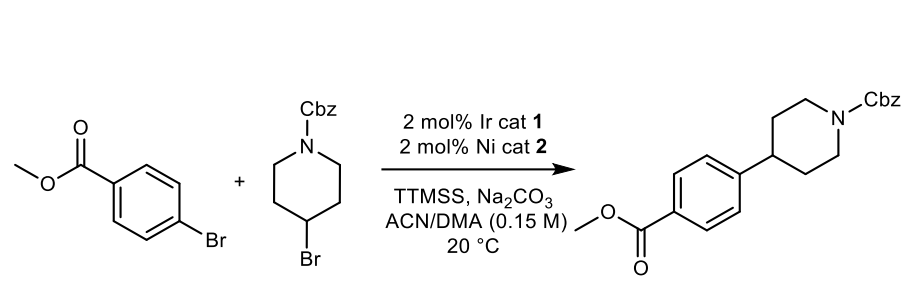

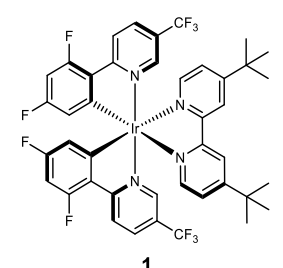

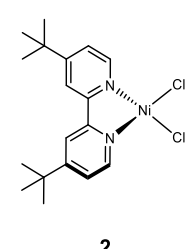

2
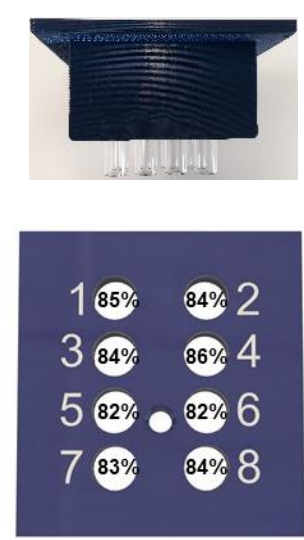

$1 \mathrm{ml}$ Vial Holder
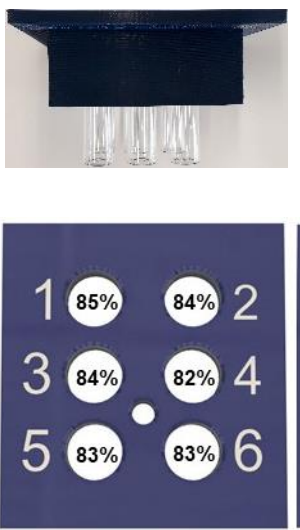

$4 \mathrm{ml}$ Vial Holder
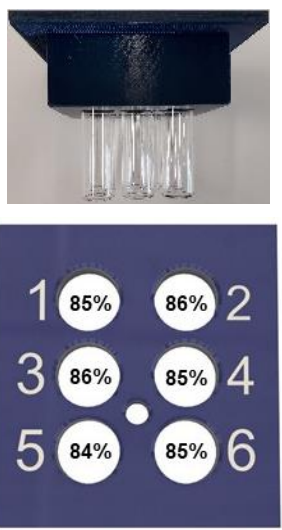

$8 \mathrm{ml}$ Vial Holder

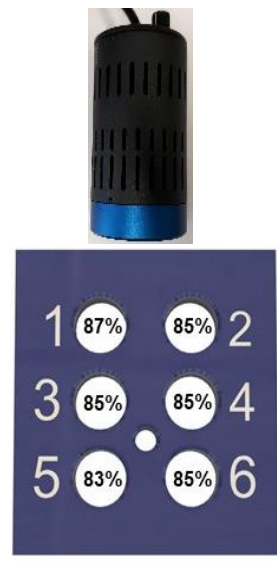

Kessil PR160L $45 \mathrm{~W} 440 \mathrm{~nm}$

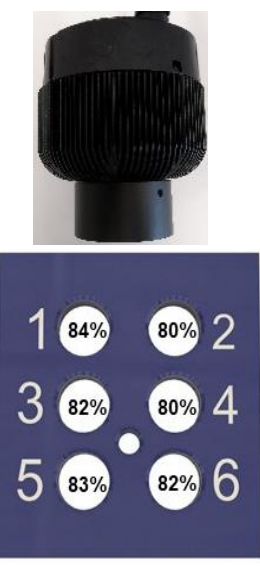

HepatoChem $30 \mathrm{~W} 450 \mathrm{~nm}$

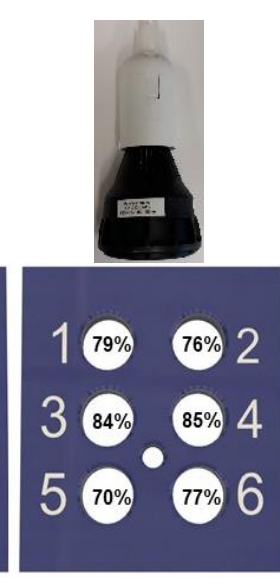

HepatoChem $18 \mathrm{~W} 450 \mathrm{~nm}$

Figure 4. a) Reproducibility of the $1 \mathrm{ml}, 4 \mathrm{ml}$ and $8 \mathrm{ml}$ vial holders with the Kessil PR160 $45 \mathrm{~W} 440 \mathrm{~nm}$ lamp, b) Reproducibility of the $4 \mathrm{ml}$ vial holders with different lamps using the optimized conditions of the cross-electrophilic reaction reported by MacMillan. The yield for each position was calculated using a HPLC calibration curve with o-terphenyl as internal standard.

Six positions are available for the 4 and $8 \mathrm{ml}$ vials and eight for the $1 \mathrm{ml}$ vials, all of which perform with the same efficiency using the Kessil PR160 45 W 440 nm lamps (Figure 4). The benchmark reaction was the $\mathrm{sp}^{2}-\mathrm{sp}^{3}$ cross-electrophile coupling method from the MacMillan group ${ }^{[13]}$ with optimized conditions. The vials can easily be removed from the reactor during the reaction (e.g. for taking a reaction control) while the LED lamps are still running, light exposure to the environment is minimal. Since reproducibility depends on the identical light intensity in each vial, all positions of the $4 \mathrm{ml}$ vial holder were tested with different lamps (Kessil PR160L $45 \mathrm{~W} 440 \mathrm{~nm}$ and HepatoChem $450 \mathrm{~nm} 18$ \& $30 \mathrm{~W}$, Figure 4). The Kessil PR160 45 W 440 nm, PR160L 45 W 440 nm and the HepatoChem $30 \mathrm{~W} 450 \mathrm{~nm}$ can be used without any restrictions. Only with the HepatoChem $18 \mathrm{~W} 450 \mathrm{~nm}$ lamp the positions 3 and 4 were faster than the other four, probably due to the more focused beam.

For larger scales, a holder for $50 \mathrm{ml}$ and $100 \mathrm{ml} \mathrm{NS} 25$ flasks and a flow unit ( $7 \mathrm{ml}$ capacity) are available (Figure 5$)$. The batch version benefits from the simple design, but it suffers from a lower light penetration, according to the Beer-Lambert law, especially in heterogeneous mixtures. Additionally, temperature gradients in the flask may arise due to a lower heat transfer.

On the other hand, flow chemistry has a better scalability due to the small reaction volume, but the set-up is usually more complex. Since most photochemical reactions do not proceed in the absence of light, no complex mixing procedure has to be taken into account. This simplifies the process: All components are combined beforehand and only a normal syringe pump is necessary (Figure 6).

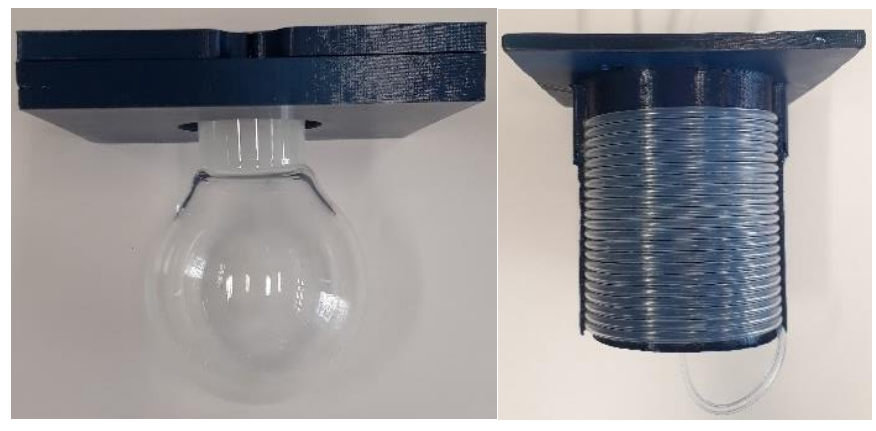

Figure 5. Picture of the flask holder (left) and the flow unit (right).

Upscaling in the batch variant was demonstrated with the crosselectrophile coupling benchmark reaction. At a $1 \mathrm{~g}$ scale in a $100 \mathrm{ml}$ flask the efficiency of the transformation was the same as in the small scale with a slightly increased reaction time $(81 \%$ yield, $3 \mathrm{~h}$ ). However, larger scales with this catalyst loading are not ideal, especially if expensive metals such as Iridium are used. Therefore, the reaction was further optimized to reduce the loading of the $\mathrm{Ir}$ catalyst to $0.1 \mathrm{~mol} \%$ (see SI for the optimization table). With these optimized conditions, a yield of $72 \%$ was isolated after $16 \mathrm{~h}$ (Scheme 1). 
<smiles>COC(=O)c1ccc(Br)cc1</smiles>

Standard conditions ( $2 \mathrm{~mol} \% \mathrm{Ir}): 81 \%, 3 \mathrm{~h}$ Optimized conditions $(\mathbf{0 . 1} \mathbf{m o l} \% \mathrm{Ir}): 72 \%, 16 \mathrm{~h}$

Scheme 1. Optimized conditions for the cross-electrophile coupling method with $0.1 \mathrm{~mol} \%$ Ir catalyst loading performed at a $1 \mathrm{~g}$ scale.

\section{Evaluating literature reactions}

The reactor was tested on several photoredox reactions, including batch, flow and photoelectrochemistry.

Batch: The arylation of aryl halides by a $\mathrm{C}-\mathrm{H}$ activation (Scheme 2) by the König group ${ }^{[14]}$ was carried out by a custom-made aluminum block setup with blue LED irradiation of the bottom side of the vials. They achieved a yield of $87 \%$ after $3 \mathrm{~h}$, whereas with the 3D-printed photoreactor the reaction time was reduced to only $15 \mathrm{~min}$ (14-fold increase) with an isolated yield of $78 \%$. In contrast to the reported conditions, low yields were obtained when the reaction mixture was carefully degassed and complete conversion was observed only in the presence of oxygen.

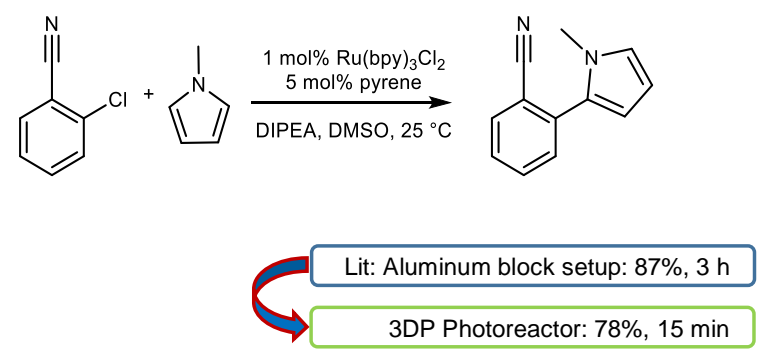

Scheme 2. Reaction conditions of the $\mathrm{C}-\mathrm{H}$ activation.

The deaminative $\mathrm{sp}^{2}-\mathrm{sp}^{3}$ cross coupling reaction (Scheme 3) was reported by the Molander group ${ }^{[15]}$ with an isolated yield of $73 \%$ after $24 \mathrm{~h}$ with a blue LED light string ring. Under high intensive LED irradiation, the yield was increased to $88 \%$ after $6 \mathrm{~h}$, which corresponds to a rate increase by a factor of 4 .<smiles>COC(=O)c1ccc(Br)cc1</smiles><smiles>Pc1cc(-c2ccccc2)cc(-c2ccccc2)[n+]1C1CCOCC1</smiles>
$\underset{\mathrm{NEt}_{3}, \mathrm{THF}_{2} 25^{\circ} \mathrm{C}}{5}$<smiles>COC(=O)c1ccc(C2CCOCC2)cc1</smiles>

Lit: LED string ring: $73 \%, 24 \mathrm{~h}$

3DP Photoreactor: $88 \%, 6 \mathrm{~h}$

Scheme 3. Reaction conditions of the deaminative cross coupling reaction.
The decarboxylative $\mathrm{sp}^{3}-\mathrm{sp}^{3}$ alkylation (Scheme 4) was reported using a classical reaction setup (Kessil lamps with fan) and with the integrated photoreactor from Merck \& Co by the MacMillan group. ${ }^{[4 a, 16]}$ With the 3D-printed photoreactor the same results as with the integrated photoreactor were obtained with a yield of $86 \%$ after $8.5 \mathrm{~h}$.
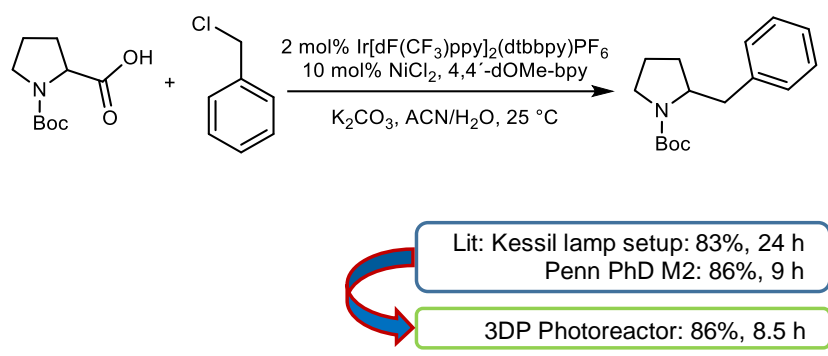

Scheme 4. Reaction conditions of the decarboxylative $\mathrm{sp}^{3}-\mathrm{sp}^{3}$ alkylation reaction.

Flow: The flow unit was tested using the $\mathrm{sp}^{2}-\mathrm{sp}^{3}$ cross coupling method using trifluoroborates and aryl bromides (Scheme 5) from the Boyd lab at Vertex Pharmaceuticals Incorporated. ${ }^{[17]}$ They used the Vapourtec E-series UV-150 device with a capacity of $10 \mathrm{ml}$. At a flow rate of $0.25 \mathrm{ml} / \mathrm{min}$ the residue time was $40 \mathrm{~min}$ and a yield of $62 \%$ was achieved. At higher light intensity the flow rate in the $7 \mathrm{~cm}$ long tube was increased to $0.35 \mathrm{ml} / \mathrm{min}$, reducing the residue time to $20 \mathrm{~min}$ with a yield of $66 \%$. The reaction was carried out using a syringe pump (Figure 6).
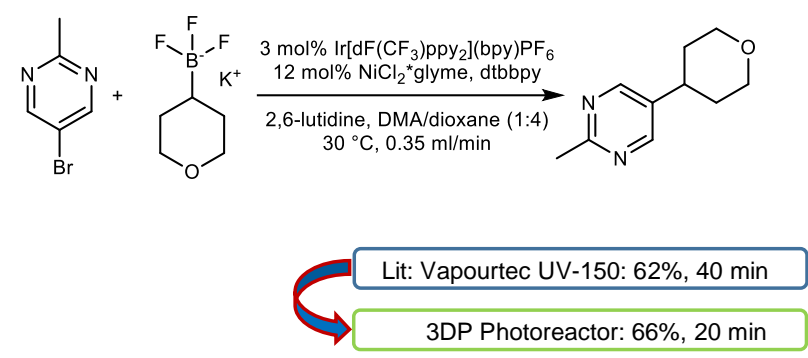

Scheme 5. Reaction conditions of the $s p^{2}-s p^{3}$ cross coupling method using trifluoroborates.

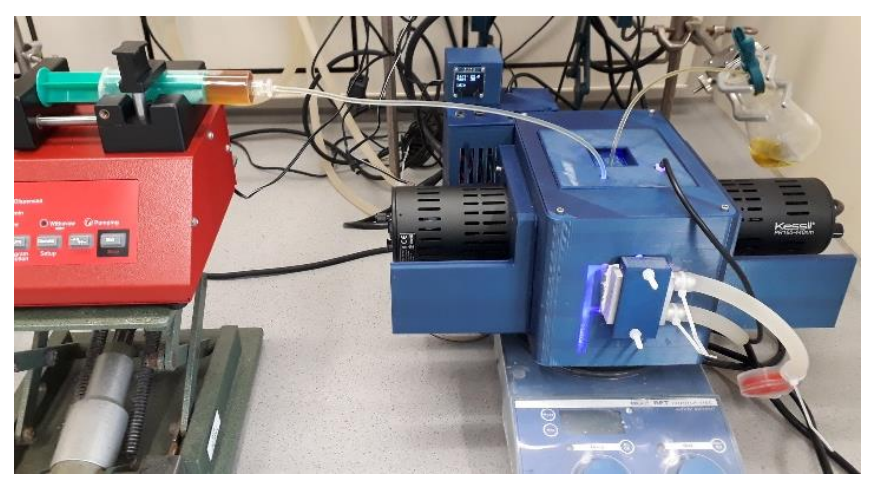

Figure 6. Picture of the reactor while performing a flow reaction with a syringe pump. 
Photoelectrochemistry: A new research field that has gained interest over recent years is the combination of synthetic photochemistry with synthetic electrochemistry to expand the spectrum of possible chemical transformations. ${ }^{[18]}$ In terms of reproducibility, this is quite a challenge, since electrochemistry also suffers from a lack of standardized equipment. However, this problem was already addressed by a collaboration between Prof. Phil Baran and IKA, to design the ElectraSyn 2.0. ${ }^{[19]}$ They also provide a module (ElectraSyn GOGO module) which allows the user to perform reactions outside the ElectraSyn (e.g. to perform reactions at different temperatures). As a result, a cover for this module was designed (Figure 7) to provide a possibility to perform photoelectrochemical transformations in a standardized manner. With the combination of both standardized reactors it was possible to reproduce the decarboxylative $\mathrm{C}-\mathrm{H}$ functionalization reaction (Scheme 6) from the Xu group. ${ }^{[20]}$ With their custommade LED/electrode setup a yield of $85 \%$ after $3.9 \mathrm{~F} / \mathrm{mol}$ was obtained. A similar yield of $83 \%$ after $3 \mathrm{~F} / \mathrm{mol}$ was isolated using both standardized reactors. However, to obtain a full conversion the loading of the oxamic acid had to be increased from 2.5 to 4 equivalents.
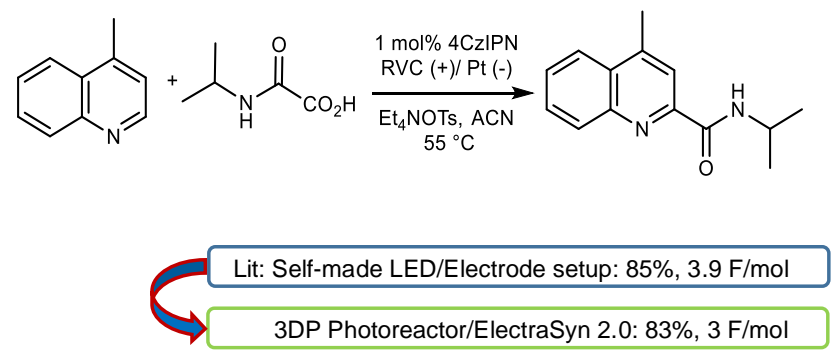

Scheme 6. Reaction conditions for the decarboxylative $\mathrm{C}-\mathrm{H}$ functionalization reaction.

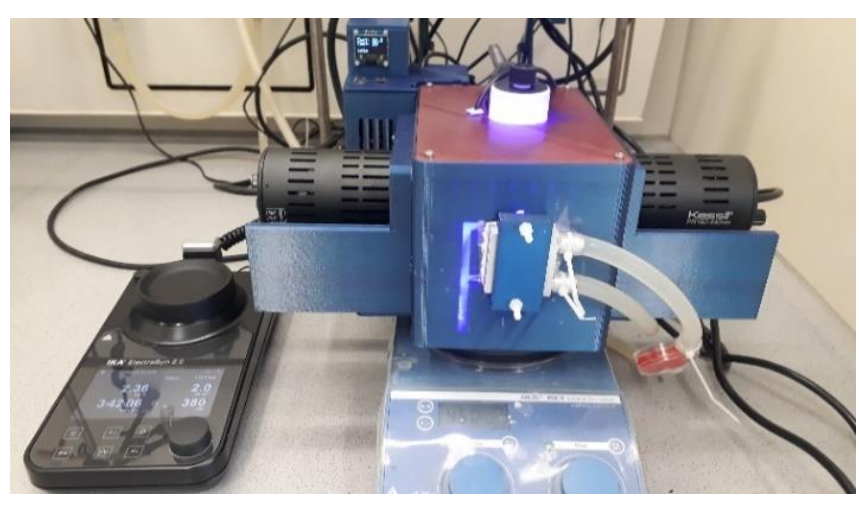

Figure 7. Performing photoelectrochemistry with the GoGo module insert.

\section{Conclusion}

A simple and easily reproducible 3D-printed photoreactor was developed, which solves various problems of photochemical setups. It allows the user to perform reactions in a standardized way at high light intensity with well-defined, commercially available LED lamps of different wavelengths. Complete temperature control over a wide range $\left(-20^{\circ} \mathrm{C}\right.$ to $\left.+80^{\circ} \mathrm{C}\right)$ has been achieved by integrating TECs into the reactor.
The flexible vial holder allows up to eight reactions to be performed simultaneously and can be easily adapted to different vial types (such as the ElectraSyn GOGO module holder). This ensures that reproducibility is not limited to a few provided vial holders. It is also designed for performing reactions on a larger scale with a flask or the 3D-printed flow unit. The reactor combines all these features while remaining small, practical and cost effective. The closed system prevents exposure to ambient light and allows safe handling without additional protective equipment.

\section{Acknowledgment}

We are grateful for the support of Dr. Stephan Zahn, Dr. Harald Weinstabl, Dr. Andreas Gollner and Prof. Nuno Maulide for valuable discussions and their ongoing support which helped to realize this project.

Keywords: Photochemistry • Electrochemistry $•$ Photoreactor $•$ 3D-printing $•$ Flow chemistry

[1] M. Baker, Nature 2016, 533, 452-454.

[2] H. E. Bonfield, T. Knauber, F. Levesque, E. G. Moschetta, F. Susanne, L. J. Edwards, Nat. Commun. 2020, 11, 804.

[3] a) J. Z. Bloh, Front. Chem. 2019, 7, 128, b) L. Buzzetti, G. E. M. Crisenza, P. Melchiorre, Angew. Chem. Int. Ed. 2019, 58, 37303747 .

[4] a) C. C. Le, M. K. Wismer, Z. C. Shi, R. Zhang, D. V. Conway, G Li, P. Vachal, I. W. Davies, D. W. C. MacMillan, ACS. Cent. Sci. 2017, 3, 647-653. b) M. N. Lavagnino, T. Liang, D. W. C. MacMillan, Proc. Natl. Acad. Sci. 2020, 117, 21058-21064 [5] C. Kerzig, O. S. Wenger, Chem. Sci. 2019, 10, 11023-11029. [6] M. Sender, D. Ziegenbalg, Chem. Ing. Tech. 2017, 89, 1159-1173. [7] a) C. P. Haas, T. Roider, R. W. Hoffmann, U. Tallarek, React. Chem. Eng. 2019, 4, 1912-1916. b) H. E. Bonfield, K. Mercer, A. Diaz-Rodriguez, G. C. Cook, B. S. J. McKay, P. Slade, G. M. Taylor, W. X. Ooi, J. D. Williams, J. P. M. Roberts, J. A. Murphy, L. Schmermund, W. Kroutil, T. Mielke, J. Cartwright, G. Grogan, L. J. Edwards, ChemPhotoChem 2020, 4, 45-51. c) S. Protti, D. Ravelli, M. Fagnoni, Photochem. Photobiol. Sci. 2019, 18, 2094-2101. d) F. Glaser, C. Kerzig, O. S. Wenger, Angew. Chem. Int. Ed. 2020 59, 10266-10284.

[8] a) B. Y. Park, M. T. Pirnot, S. L. Buchwald, J. Org. Chem. 2020 85, 3234-3244. b) H. A. Sakai, W. Liu, C. C. Le, D. W. C. MacMillan, J. Am. Chem. Soc. 2020, 142, 11691-11697. c) X. Zhang, D. W. C. MacMillan, J. Am. Chem. Soc. 2016, 138, 1386213865 .

[9] a) G. Goti, B. Bieszczad, A. Vega-Penaloza, P. Melchiorre, Angew. Chem. Int. Ed. 2019, 58, 1213-1217. b) C. Verrier, N. Alandini, C. Pezzetta, M. Moliterno, L. Buzzetti, H. B. Hepburn, A. Vega-

Peñaloza, M. Silvi, P. Melchiorre, ACS. Catal. 2018, 8, 1062-1066. a) http://www.pennphd.com/product/5, 29.11.2020. b) http://www.hepatochem.com/photoreactors-leds-accessories/, 29.11.2020. c) http://www.thalesnano.com/products-andservices/photocube/, 29.11.2020. d) http://www.pacer.co.uk/casestudies/medical-case-studies/led-illuminator.html, 29.11.2020. e) http://www. luzchem.com/ProductList.php?product line ID=1, 29.11.2020. f)

http://www.luzchem.com/ProductList.php?product line ID=11, 29.11.2020.

A. Ambrosi, M. Pumera, Chem. Soc. Rev. 2016, 45, 2740-2755

T. Guclu, E. Cuce, Journal of Elec. Materi. 2019, 48, 211-230.

P. Zhang, C. C. Le, D. W. MacMillan, J. Am. Chem. Soc. 2016, 138, 8084-8087.

a) I. Ghosh, R. S. Shaikh, B. Konig, Angew Chem. Int. Ed. 2017, 56, 8544-8549. b) M. S. Coles, G. Quach, J. E. Beves, E. G. Moore, Angew. Chem. Int. Ed. 2020, 59, 9522-9526.

[15] J. Yi, S. O. Badir, L. M. Kammer, M. Ribagorda, G. A. Molander, Org. Lett. 2019, 21, 3346-3351.

[16] C. P. Johnston, R. T. Smith, S. Allmendinger, D. W. C. MacMillan, Nature 2016, 536, 322-325.

[17] T. J. DeLano, U. K. Bandarage, N. Palaychuk, J. Green, M. J. Boyd, J. Org. Chem. 2016, 81, 12525-12531.

[18] J. P. Barham, B. Konig, Angew. Chem. Int. Ed. 2020, 59, 1173211747. 
[19] a) M. Yan, Y. Kawamata, P. S. Baran, Angew. Chem. Int. Ed. 2018, 57, 4149-4155. b) http://www.ika.com/de/Produkte-LabEq/Electrochemistry-Kit-csp-516/, 29.11.2020.

[20] X. L. Lai, X. M. Shu, J. Song, H. C. Xu, Angew. Chem. Int. Ed. 2020, 59, 10626-10632. 
